\title{
Interleukin-2 and other cytokines in candidiasis: expression, clinical significance, and future therapeutic targets
}

\author{
Carmen Rodríguez-Cerdeira ${ }^{1,2,3} \bowtie$, Miguel Carnero-Gregorio ${ }^{2,4}$, Adriana López-Barcenas ${ }^{3,5}$, Gabriella Fabbrocini³,6, \\ Elena Sanchez-Blanco33, Alfonso Alba-Menendez7, Roberto Arenas Guzmán
}

\begin{abstract}
Susceptibility to Candida spp. infection is largely determined by the status of host immunity, whether immunocompromised/immunodeficient or immunocompetent. Interleukin-2 (IL-2), a potent lymphoid cell growth factor, is a four-a-helix bundle cytokine induced by activated T cells with two important roles: the activation and maintenance of immune responses, and lymphocyte production and differentiation. We reviewed the roles of cytokines as immune stimulators and suppressors of Candida spp. infections as an update on this continuously evolving field. We performed a comprehensive search of the Cochrane Central Register of Controlled Trials, Medline (PubMed), and Embase databases for articles published from March 2010 to March 2016 using the following search terms: interleukins, interleukin-2, Candida spp., and immunosuppression. Data from our own studies were also reviewed. Here, we provide an overview focusing on the ability of IL-2 to induce a large panel of trafficking receptors in skin inflammation and control T helper (Th) 2 cytokine production in response to contact with Candida spp. Immunocompromised patients have reduced capacity to secrete Th1-related cytokines such as IL-2. The ability to secrete the Th1-related cytokine IL-2 is low in immunocompromised patients. This prevents an efficient Th1 immune response to Candida spp. antigens, making immunocompromised patients more susceptible to candidal infections.
\end{abstract}

Keywords: interleukin-2, Candida albicans, Candida spp., cytokines, immunosuppression

Received: 25 September 2017 | Returned for modification: 27 November 2017 | Accepted: 1 December 2017

\section{Introduction}

The immune system protects hosts against attack by microorganisms. The other functions of the immune system include monitoring and killing aberrant cells. The elimination of disease differs from the elimination of infection and, to control infection with minimal tissue damage, the immune system frequently controls pathogen growth without full clearance or sterility. One mechanism for this is the release of various cytokines.

Over the past years, the understanding of the host's protective mechanisms against Candida has advanced, including understanding of recognition of the fungus by the host as well as the host's innate and adaptive effector mechanisms to combat the fungus. Interleukin-2 (IL-2) is an important cytokine that mainly acts on $\mathrm{T}$ cells, promotes their proliferation, and modulates the differentiation of some $\mathrm{T}$ cell subsets (1).

IL-2 is primarily produced by T helper (Th) 1 cells, although cytotoxic T (Tc) cells can produce small amounts of this cytokine. It is a growth factor, previously called the $\mathrm{T}$ cell growth factor. It is also a B cell growth factor that promotes proliferation of B cells. IL-2 is required for proliferation and differentiation of T cells. It is an activator of both natural killer (NK) cells and monocytes. The professional antigen-presenting cell (APC) presents antigen peptide to the $\mathrm{T}$ cell receptor, and its surface $\mathrm{B} 7$ ligates $\mathrm{T}$ cell CD28, activating the $\mathrm{T}$ cell to produce IL-2 and its receptor (IL-2R). The cytokine acts in an autocrine fashion. The cell divides and differentiates into an effector $\mathrm{T}$ cell, which no longer requires a signal for its effector function. At the termination of the immune re- sponse, cytotoxic T-lymphocyte antigen 4 (CTLA-4) replaces CD28 and downregulates $\mathrm{T}$ cell function via reducing the expression of IL-2 receptor (2) (Fig. 1).

As such, IL-2 also plays an important role in the context of candidiasis, and therefore this review treats a relevant aspect in infection research and fungal disease.

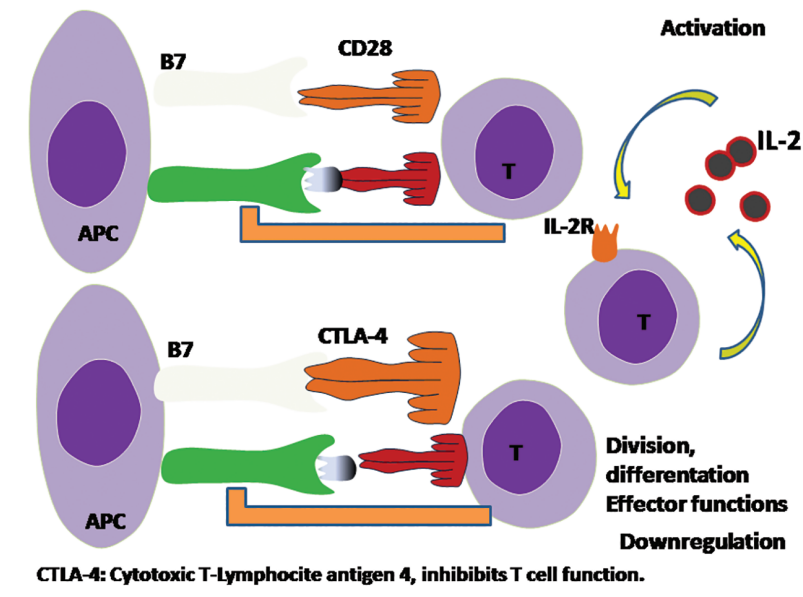

Figure 1 | Activation: co-reception of both signals, TCR and CD28, from the surface of a professional APC activates the T cell to produce IL-2 and its receptor (IL-2R). The cell divides and differentiates into an effector T cell, which no longer requires signal 2 for its effector function. Downregulation: at the termination of the immune response, CTLA- 4 replaces CD28 and downregulates T cell function. $\mathrm{B} 7 \mathrm{p}$ peripheral membrane protein found on activated antigen presenting cells (APC), TLC = Toll-like receptor. 
Individuals without a weakened immune system can also suffer from these infections. It is well known that fungi are a major target for the immune system; they are made visible by the expression of molecular patterns or signatures associated with the pathogen.

We now observe the role of innate and adaptive immunity in eliminating fungal infections and how a disproportionate or inadequate immune response may decrease the host's ability to eliminate fungi (1).

T cells can be stimulated to grow in the presence of both classes of molecules, antigens or mitogenic lectins (such as phytohemagglutinin or concanavalin A), to secrete a detectable amount of IL-2, which is a strong factor in lymphoid cell growth. IL-2 has a four-a-helix bundle structure and is generated by activated $\mathrm{T}$ cells, particularly the CD4+ Th cell population.

By activating regulatory $\mathrm{T}$ cells (Tregs) against cytotoxic effector cells, IL-2 induces both immunosuppressive and immunostimulatory effects (3). IL-2 is vital for self-antigen tolerance, as shown by the autoimmune phenotype of IL-2-knockout mice (4) (Fig. 2). Mehra et al. (5) showed that the IL-2 promoter is inactive in naive cells, but binding with chromatin-remodeling complexes, histones, and transcription factors such as AP-1, NF- $\mathrm{kB}$, NFAT, and OCT-1 facilitates the rapid onset of IL-2 promoter activity. IL-2 expression is controlled by the strength and duration of T cell receptor (TCR) signaling, co-stimulation, and rate of degradation of IL-2 mRNA.

To investigate the individual contributions of tumor necrosis factor (TNF)-receptor 1 and TNF-receptor 2 to IL-2 expression, Miller et al. (6) generated 5C.C7TCRRag2-/- IL-2-GFP reporter mice lacking both TNF-receptor 1 and TNF-receptor 2 expression, and examined IL-2 production at the level required to enable promoter activity. They showed that, in addition to promoting the genera- tion of FoxP3+Tregs, TNF-receptor 2 hinders Th17 cell differentiation by promoting IL-2 expression (Fig. 3). Suppression of CD4+ T cell-intrinsic TNF-receptor 2 was sufficient to encourage Th17 differentiation under Th17-polarizing conditions (5).

It is well known that, in the context of Candida, T cells producing IL-17 (Th17) cells are the protective Th subset, as supported by the fact that the majority of Candida-specific memory Th cells in healthy individuals that have been exposed to the fungus belong to the IL-17-producing subset. Th17 are considered a new line of effector $\mathrm{T}$ cells. They present a great heterogeneity that affects their requirements, migratory capacities, and role in defense against pathogens (7).

To exert their defense mechanism, memory and effector T cells must migrate to chemokine-containing tissues due to the inflammatory/infectious process. The receptors for these chemokines determine the different subpopulations of $\mathrm{T}$ lymphocytes. Thus, for example, memory T lymphocytes have CCR7 receptors, interferon (IFN)-y-producing Th1 cells have CXCR3 and CCR5 receptors, and Th2 cells that produce IL-4, IL-5, and IL-13 have CCR3, CCR4, and CRTH2 receptors. Th17 cells have been discovered in

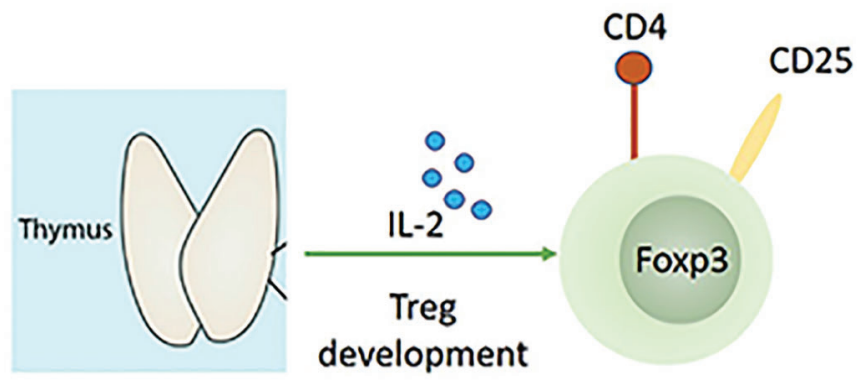

Figure 3 | Naturally occurring Tregs (CD4+CD25+Foxp3+ cells) derived from thymus. IL-2, cell contact-dependent inhibition. Foxp3 is required for Treg development. Foxp3 = Forkhead box P3.

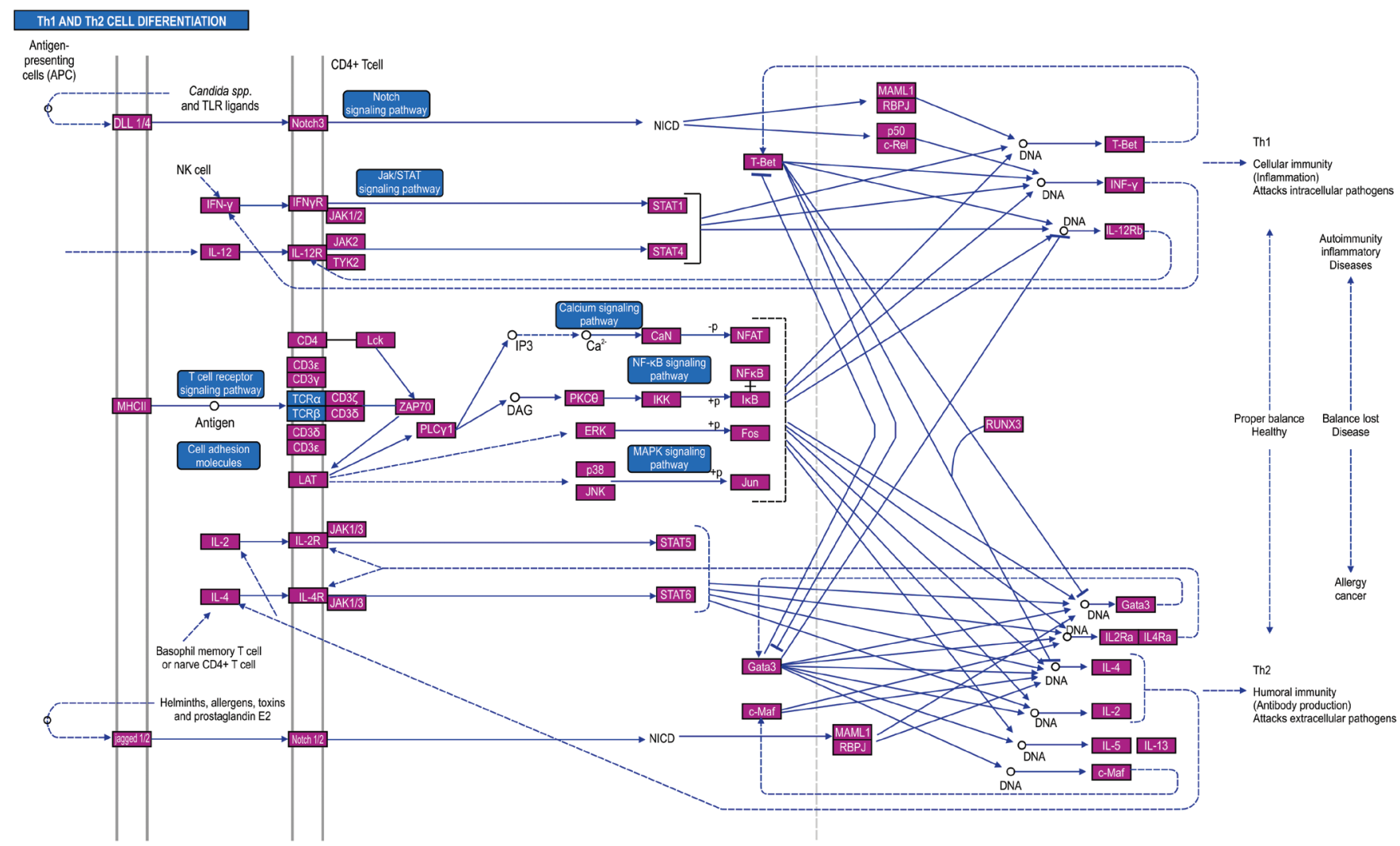

Figure 2 | Immunity to Candida spp. is orchestrated by separate lineages of effector Thelper (Th) cells, which differentiate from naive CD4+ precursor cells in response to cues provided by antigen presenting cells (APC) and include Th1 and Th2. Th1 cells are characterized by the transcription factor T-bet and signal transducer and activator of transcription (STAT) 4, and the production of IFN-gamma. The balance between Th1/Th2 subsets determines the susceptibility to disease states. However, an overactive Th1 response can lead to autoimmunity (4). 
mice, and their membrane receptors were found to be different from those seen in other $\mathrm{T}$ lymphocyte subpopulations. There is a Th17 population expressing RORC mRNA that has CCR6 receptors, which is a distinguishing feature in those cells involved in the response to fungal infections. Expression of the CCR6 receptor is an important feature in the differentiation of Th17 cells. Certain CCR6+ cells also express the CCR1o receptor; however, they do not produce IL-17, but IL-22, a Th17-related cytokine. This variety of cells, called Th22, is considered a subtype of Th17 and plays an important role in processes of homeostasis and inflammation (8).

For the expression of RORyt and CCR6 in Th17, participation of IL-6, IL-1 $\beta$, and IL-23 is required. However, TGF- $\beta$ is an inhibitor of this process in human Th17 cells, unlike what occurs in mice.

On the other hand, research by Gil et al. (9) showed that the interaction of $C$. albicans with TLRs is a complex process because TLRs can collaborate with other pattern recognition receptors (PRRs) and the expression of fungal ligands associated with the surface depends on the strain and the morphotype (yeasts or hyphae), thus defining the final induced response (Th1/Th2/Th17; Fig. 4).

In studies carried out by Ju et al. (10) on mice with mutated IL2, Treg generation occurred due to compensation by IL-4 and IL-7. In these mice, weak inflammation of the lungs, but not the skin, was observed. To eliminate the effect of those Tregs activated by other pathways, a series of assays were performed with IL-2 and FoxP3 double-mutant mice (Sf/IL-2), which prevented the activation and proliferation of Tregs, as well as the role of IL-2 in the inflammatory processes of the skin and lung. On the basis of these findings, the following conclusions can be derived:

1. IL-2 is not required for the in vivo expression of cytokine genes from Th2 lymphocytes but for the production of Th2 cytokines upon T-lymphocyte activation.

2. Cytokines regulating the expression of trafficking receptor genes in Th subsets in Sf mice are IL-2, IL-4, and IFN-y (Fig. 5).

3. Inhibition of genes for receptors that were specifically regulated by IL-4, signal transducer and activator of transcription 6 (STAT-6), and IFN-y was not sufficient to prevent inflammation of the skin and lungs as in Sf/IL-2 mice.

IL-2 is mainly produced by activated CD4+ T cells. The binding of a cytokine to its appropriate receptor sets off a cascade that leads to induction or inhibition of transcription of a number of cytokine-regulated genes. The a chain binds IL-2 with low affinity, the CD122 (IL-2Rß)/CD132 y-chain complex with intermediate affinity (Kd, 9-10 M), and the CD122/CD132/CD25 (IL-2Ra) receptor complex with high ligand-specific affinity (Kd, 10-11 M). The high affinity IL-2 consists of the IL-2Ra chain, the IL-2R $\beta$ chain, and the IL-2Ry chain; these complexes respond to IL-2 doses of $\sim 1 \mathrm{nM}$ and $\sim 10 \mathrm{pM}$, respectively (11), to activate the downstream STAT $_{5}$, mitogen-activated protein kinase (MAPK), and phosphatidylinositol-4,5-bisphosphate 3-kinase ( $\mathrm{PI}_{3} \mathrm{~K}$ ) signaling cascades (13). Consumption of IL-2 in the periphery by CD4+ effector T cells (Teffs) or Tregs contributes to the control of Th cell differentiation and the suppression of Treg function, respectively (12) (Fig. 6).

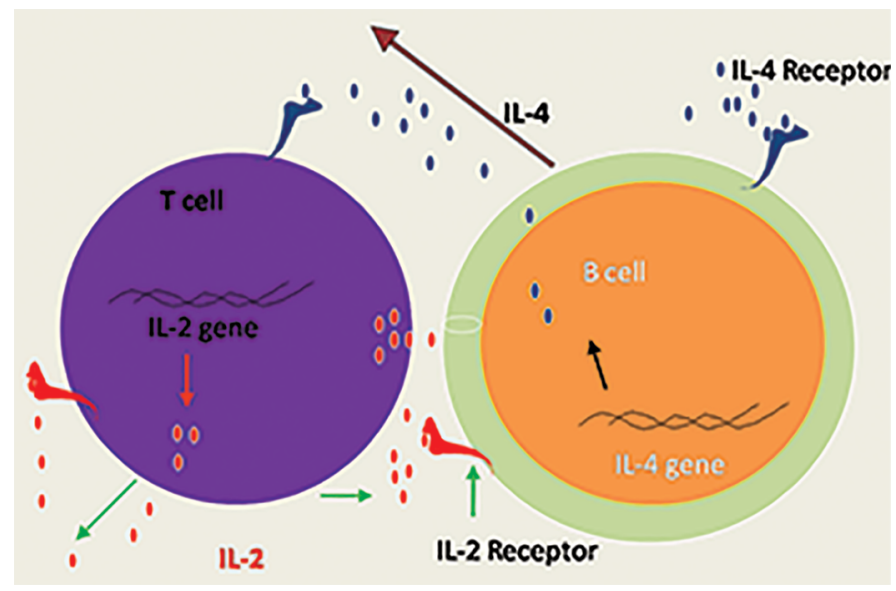

Figure 5 | Cytokine network at a local level. Cytokines are produced in response to various stimuli, such as antigen receptors and cytokine/chemokine receptors. Cytokines have various modes of action: autocrine, in which the cytokine acts on the same cell that produced it (IL-2 for T cell activation); paracrine, in which it acts on nearby cells (T cells help for B cells); and endocrine, in which it acts on distant cells (inflammatory cytokines).

$\mathrm{IL}=$ interleukin.

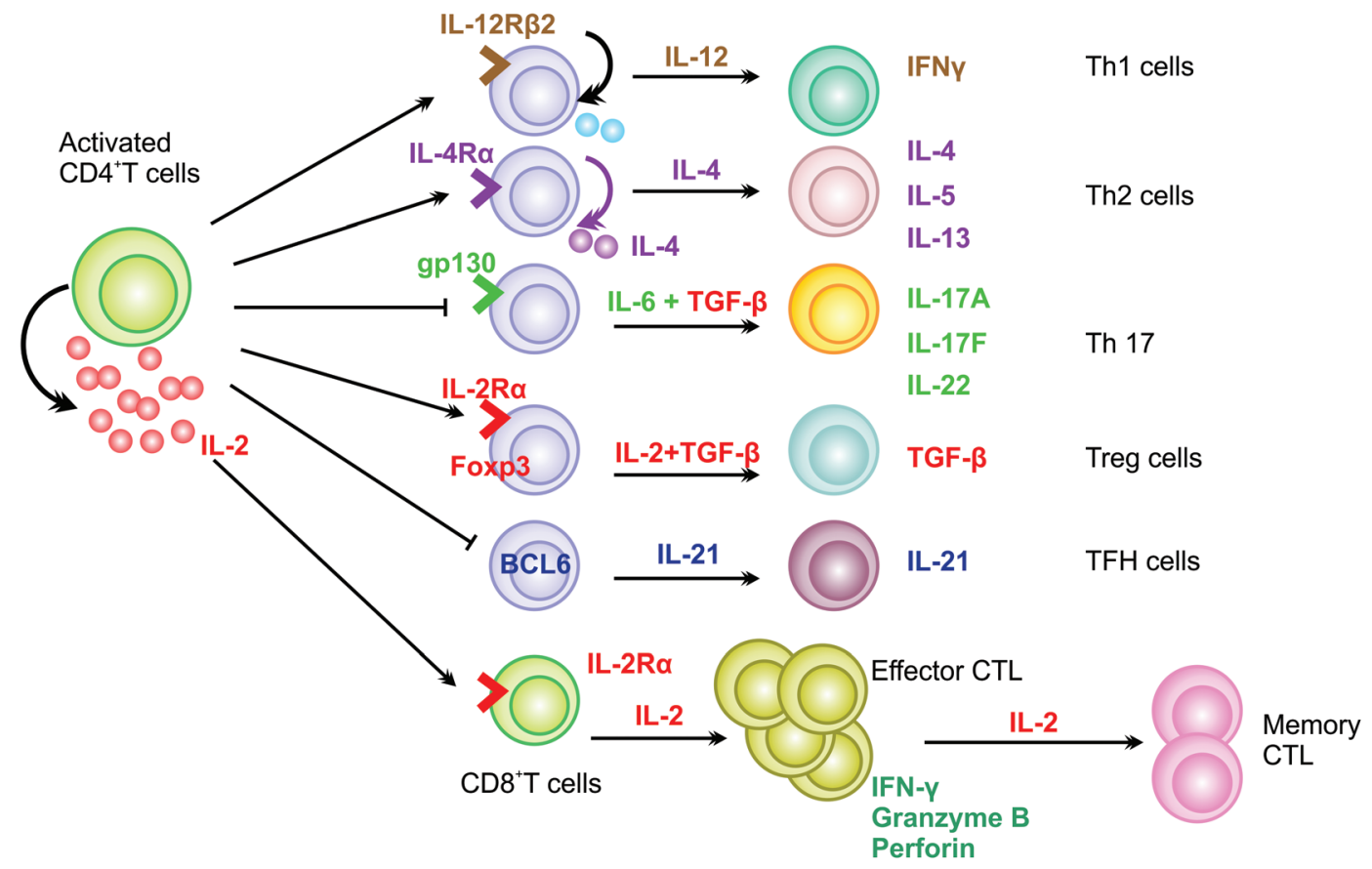

Figure 4 | Shown is the induction by IL-2 of IL-12Rß2 to promote Th1 cell differentiation, of IL-4Ra to promote Th2 cell differentiation, and of IL-2Ra to promote Treg cell differentiation.

$\mathrm{CTL}=$ cytotoxic $\mathrm{T}$ lymphocytes, Gp $130=$ glycoprotein 130 , TFH $=\mathrm{T}$ cell follicular helper, BCL6 $=\mathrm{B}$ cell lymphoma 6 protein, $\mathrm{TGF} \beta=$ transforming growth factor beta, Foxp3 = Forkhead box P3, IL = interleukin. 


\section{Structures of the IL-2 and IL-4 receptors}

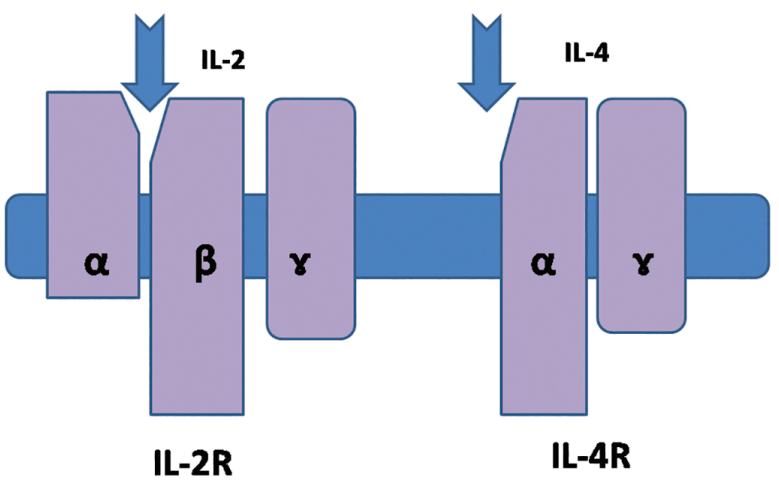

Figure 6 | The high-affinity IL-2 receptor is formed by three polypeptide chains, of which the $\alpha$ and $\beta$ chains bind to the cytokine, whereas the $y$ chain is involved in signaling to the cell. The IL- 4 receptor shares the $\gamma$ signaling chain but this chain specifically recognizes IL-4.

$\mathrm{IL}=$ interleukin.

As Yamakazi et al. (13) showed, Candida spp. are the major cause of systemic nosocomial fungal infections, particularly for patients in intensive care receiving antibiotics or undergoing immunosuppressive therapy or surgery (13). Approximately $20 \%$ of infections found in intensive care unit patients and $11 \%$ of all bloodstream infections are caused by Candida spp., among which C. albicans remains the most frequently isolated yeast species (13).

The use of pro-inflammatory, anti-inflammatory, regulatory, or suppressor cytokines as a means of modulating immunity against Candida spp. has received much attention in recent years (11, 13). Numerous studies in this area have focused on the use of cytokines and granulocyte macrophage colony-stimulating factor in conjunction with drug-based antifungal treatment.

A better understanding of the host's immune response in fighting fungal infection can provide useful clues as to how new targets and agents might be developed to treat those diseases in the future. Here, we provide an overview of the key studies on immune responses in Candida spp. infections and highlight the promising potential of targeting $\mathrm{T}$ cells as an immunotherapy approach for treating candidiasis and other fungal infections.

\section{Mucocutaneous candidiasis}

Candida is a commensal microorganism with pathogenic potential. PCR-based identification has shown that Candida spp. colonizes the gastrointestinal, genitourinary, and respiratory mucosa, as well as the skin (14). However, the isolation of Candida from these tissues does not always imply the presence of infection. The transition between colonization and mucosal invasion or the spread of infection depends largely on the host's immune response. The PCR technique using P-I and P-II is very useful for molecular typing of Candida albicans, whereby the similarity of genotypes isolated from the same individual can be verified and this is not dependent on the infectious or non-infectious phase or on the location of Candida in the body (15). C. albicans induces a host immune response, the efficacy of which depends on the balance between the CD4+Teff and Tregs responses. Through several cell cultures, Whibley et al. (15) verified the formation of both Tregs and Teffs, indicating that $C$. albicans induced the proliferation of Foxp3 + T cells from both other preexisting Tregs and Teffs. This highlights the importance of Foxp3+Treg and Teff activation, which impedes pathogen eradication, and the significance of Treg-mediated immune suppression, which can shift and dictate the tempo of some persistent infections.
As Kruger et al. (16) stated, although different forms of candidiasis are discussed, a clear distinction of invasive and superficial is necessary. It does seem important, however, to make this distinction because the host defense mechanisms largely differ between the two, with myeloid cells (neutrophils and monocytes) being critical for protection from systemic infection, whereas superficial infections are instead combated by $\mathrm{T}$ cells and/or epithelial defenses such as antimicrobial peptides.

As in other infections, in C. albicans infections as well, mediators of the inflammatory response lead to an immune response (Fig. 7). Inflammasome-derived IL-1 $\beta$ stimulates the production of IFN-y through Th1 cells, as well as IL-17A from Th17 cells. Sharma et al. (17) demonstrated that IL-2 might act as a suppressor or activator of immunity. Therefore, IL-2 has a pro-inflammatory function whose effect can be inhibited by antigen-specific Tregs.

Ryan et al. (18) found that defects in cell-mediated immunity favor Candida infection and the appearance of mucocutaneous candidiasis (CMC). Type 1 autoimmune polyendocrinopathy syndrome (APECED) occurs in CMC patients with a mutation in an autoimmune regulatory gene (AIRE).

As described by Lévy et al. (19), CMC has a genetic component with various inheritance patterns by mutations in genes that affect immune function. One of the genes that produces CMC, as well as recurrent Staphylococcus infections, is IL-17RA. This study was conducted on 21 patients from various countries in Africa, Asia, and South America. Analyzing the genome of healthy relatives, they were observed to be either heterozygous or homozygous for the wild-type allele, which is consistent with an autosomal recessive (AR) inheritance. A total of 12 mutant allelic variants (including p.D387 and p.Y591Sfs * 29) were found that were not previously recorded in any database.

All of the patients showed a total IL-17RA deficiency, with lack of cellular response to IL-17A and IL-17F in fibroblasts, as well as IL${ }_{17} \mathrm{E}$ and IL-25 in peripheral blood mononuclear cells (PBMCs). This lack of cellular response to these molecules causes susceptibility to recurrent infections by Staphylococcus aureus and C. albicans.

The identification of several groups of patients suffering from CMC that have defects in genes of the IL-17 pathway was studied by Puel et al. (20). Certain CMCs are associated with primary T cell immunodeficiencies caused by mutations in certain genes that affect this lymphocyte population, such as CD25, DOCK8, TYK2, TCR-a, ORAI1, MST1, and IRF8 deficiencies or NEMO or IkBa disorders.

In the case of syndromic CMC, hyper IgE syndrome (HIES), an autosomal dominant (AD) disease, has a low proportion of Th17, as well as AR deficiencies of IL-12p40 and IL-12Rß1. The same low proportion of IL-17-producing T cells was seen in AR CARD9, which participates in the regulation of receptors that recognize $C$. albicans. Patients with autoimmune AR disease polyendocrinopathy syndrome type I (APS-I) have low levels of IL-17A, IL-17F, and/or IL-22.

Okada et al. (21) distinguish two groups of CMC: syndromic CMC (SCMC) and CMC diseases (CMCD). CMCD encompasses CMCs that are not related to genes that cause combined immunodeficiency or those that cause SCMC.

Among the mutations that can cause SCMC, the following were found:

- HIES with AD inheritance and mutation of the STAT3 gene, responsible for signal transduction for IL-6, IL-10, IL-17, and IL22, among other things;

- APS-1 syndrome with AR inheritance, which develops auto- 
Inflammatory response to Candida spp.

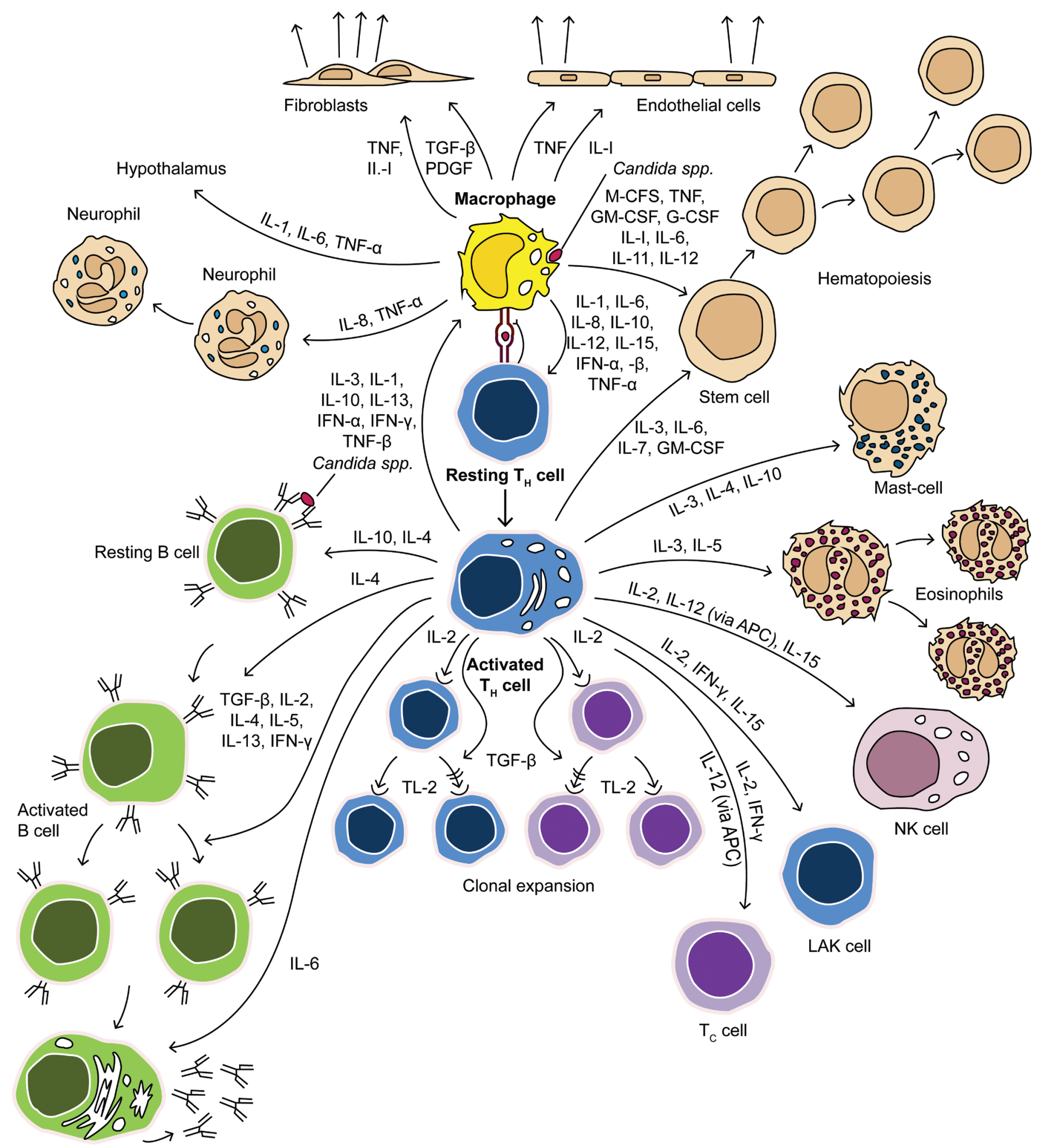

Plasma cell

Figure 7 | The immune system recognizes the presence of pathogens (Candida spp.) through several proteins that bind to molecules secreted by the pathogen or carried on their surface. The cell responsible for these immune responses have distinct roles in the immune system and communicate with other immune cells by cytokines.

LAK cell = lymphokine-activated killer cells, IL = interleukin, TNF = tumor necrosis factor, NK cell = natural killer cell.

antibodies to IL-17A, IL-17F, and/or IL-22;

- CARD9 deficiency with AR inheritance. This molecule binds to BCL10, Malt, and NEMO to induce proinflammatory cytokine production through NFkB.

- Deficiencies in IL-12Rß1 and IL-12p40, both with AR inheritance. They are part of the IL-12 / IL-23 receptors and IL-12 / IL-23 cytokines, respectively. These genes participate in the differentiation processes of Th1 lymphocytes and the expansion of IL17-producing Th17 lymphocytes.

- STAT1 gain-of-function with AD inheritance. Defects in this gene cause hyperphosphorylation of STAT1; in addition, these patients present defects in B lymphocytes and low levels of 
IgG2 and IgG4. The mechanism of low IL-17 production by $\mathrm{T}$ cells in this type of patients is unclear, but it is believed that increased STAT1 activity could inhibit the development of IL-17 T lymphocytes. On the other hand, it could also affect IL-21- and/ or IL-23-induced STAT3 activity.

- Deficiency in RORyT with AR inheritance. RORyT plays an important role in the development of Th17 and in the expression of CCR6 receptor.

The IL-17 cytokine family consists of six members (from IL-17A to IL-17F), whereas the IL-17 receptor family consists of five members (from IL-17RA to IL-17RE). IL-17 signaling plays an important role in mucocutaneous immunity to Candida. Within the molecular defects of the CMCD, one finds the following:

- Deficiency of IL-17F with AD heredity;

- Deficiency of IL-17RA with AR inheritance;

- Deficiency of IL-17RC with AR inheritance; and

- Deficiency of ACT1 with AR inheritance.

According to Eslami et al. (22), one of the major barriers to Candida infection is IL-17-mediated immunity. Various dominant and recessive autosomal mutations (HIES, APECED, IL-12p40 / IL$12 \mathrm{R} \beta 1$, or CARD9 deficiency) produce an increase in the predisposition to infection by this fungus due to impaired IL-17-mediated immunity. Another type of AD mutation that is described as the genetic etiology of CMC disease is the gain of STAT1 function (STAT1 GOF). In that article the authors describe the clinical case of a 33-year-old man affected from childhood by recurrent episodes of candidiasis on the skin, mucous membranes, and nails. Other infectious episodes were recorded during adolescence and adulthood. His son died at 6 months due to a sepsis process. $C$. albicans resistant to azoles was isolated from the patient's oral lesions. Flow cytometry techniques showed that blood immunoglobulins and the CD4/CD8 ratio were normal. Laboratory tests showed an impaired response of lymphocytes to $C$. albicans. Genetic testing indicated a mutation in the STAT1 gene. The results of the tests showed that the patient had a STAT1 GOF mutation. The episodes of recurrent candidiasis coincided, but this patient did not suffer from the serious viral or mycobacterial infections suffered by people with this type of mutation. These data suggest that STAT1 GOF mutations in heterozygosis may be present with a wide variety of clinical signs and symptoms; hence, more cases should be studied to determine a genotype-phenotype relationship in this type of mutation (22).

DCs produce IL-2 following initiation of actin-mediated phagocytosis, which leads to Src and Syk kinase activation, Ca2+ mobilization, and calcineurin-dependent activation of NFAT, the master transcription factor regulating IL-2 expression.

Khameneh et al. (23) demonstrated that DC-derived IL-2 promotes the adaptive immune response to alumina immunization; our results suggest that adjuvants targeting DC to increase IL-2 production could increase vaccine efficacy. DC-derived IL-2 as a key mediator of alum adjuvanticity in vivo and the Src-Syk pathway DC-derived IL-2 as a key mediator of alum adjuvanticity in vivo and the Src-Syk pathway is a key point for the design of new adjuvants.

In addition, Sancho and Reis e Sousa (24) state that DCs provide an important source of IL-2 and that DC-derived IL-2 is particularly strongly induced in response to fungal cell wall components that signal via pattern recognition receptors of the C-type lectin receptor family.

According to Whitney et al. (25), certain Candida pattern recognition receptors (PRRs) belong to the Toll-like (TLR) or C-type lectin receptor (CLR) families. Within the TLRs is MyD88, which transduces the signal of IL-1 and IL-8, important in innate antifungal immunity. CLR activates a tyrosine kinase (Syk) that triggers NFkB-dependent transcription signals through CARD9. This work shows that the coordination of the innate immune system against the systemic infection of $C$. albicans is dependent on Syk expression in CD11c + cells. If the Syk mediator is not present, there is no release of IL-23p19, which is imperative for producing GM-CSF by NK cells in the kidney. Susceptibility in CD11c $\Delta$ MyD88 mice did not increase. In addition, in evaluating the neutrophil population recruited in the kidney, in CD11c $\Delta$ Syk mice it was observed that its operation was not normal, although its number did not change. When levels of certain molecules were compared in control mice and in CD11c $\Delta$ Syk mice, there was no GM-CSF production in the latter. If exogenous treatment with GM-CSF was given, the C. albicans burden decreased in CD11c $\Delta$ Syk mice, whereas it had no effect on control mice or CD11c $\Delta$ MyD88 mice. Transferring NK cells from control mice to $C D 11 \mathrm{c} \Delta$ Syk mice prior to infection, the fungal load was not affected, but, if infected NK cells were transferred from infected control mice to infected CD11c $\Delta$ Syk mice, the fungal load decreased. Kidney NK cells were found to have IL-23R receptors, but their expression was not regulated in $\mathrm{CD} 11 \mathrm{c} \Delta \mathrm{Syk}$ mice. The same was observed in IL-23p19 KO mice, and so it was concluded that this subunit of IL-23 is required for the regulation of IL-23R receptors that stimulate the production of GM-CSF in NK cells. Production of IL-23p19 is stimulated by Syk. The discovery of this mechanism of cellular signaling will allow the development of new therapeutic targets for the disseminated infection of $C$. albicans (25).

Dendritic cells (DCs) have previously been named on the basis of cell morphology, expression of specific markers, and select functional attributes such as the ability to migrate to $\mathrm{T}$ cell areas of secondary lymphoid organs and activate T lymphocytes. However, these properties are not qualitative and may change under conditions of infection. However, the nature of the pathogen could dictate the type of cytokine produced by DC subsets, allowing them to prime distinct types of immune responses. Overall, DCs have a significant quality of being easily shaped or molded to respond to infection and direct adaptive immunity (26).

DCs produce cytokines such as IL-2, which are important for the generation of immune responses mediated by Th1 lymphocytes. In a study on CMC patients, including those with and without APECED, Ryan et al. found that CMC patients had altered DC function, albeit in a different way. Compared with the control group, increased production of IL-6 and decreased IL-23 in response to Candida were only observed in non-APECED patients. Hyperactivation of DCs was only observed in APECED patients. Both impaired DC maturation and overproduction of certain LPSinduced cytokines (IFN-y, IL-2, TNF-a, IL-5, and IL-13) were similar in APECED and non-APECED patients (18).

Lilic et al. (27) demonstrated that CMC patients in contact with $C$. albicans antigens exhibit decreased production of Th1 cytokines, including IL-2 and IFN-y, and increased production of anti-inflammatory cytokines such as IL-10. The immune response in CMC patients with impaired production of type 1-inducing cytokines (perhaps in response to macrophages or DC defects) is unable to mount protective cell-mediated responses, leading to a failure to clear Candida. Similar results were obtained by Bahri et al. (28) for C. parapsilosis.

Khosravi et al. (29) demonstrated the responses produced by lymphocytes and quantified cytokine secretion from PBMCs in 
samples from CMC patients. They highlighted that most patients had a low proliferative response to $C$. albicans antigens (92.3\%). PBMCs from CMC patients showed lower levels of the Th1 cytokines IL-2 (78.5-59.8 pg/ml) and IFN-y (115.1-43.3 pg/ml) compared to those in the control group.

Richardson et al. (30) evaluated the roles of DCs in C. albicans infection. DCs are immune cells specialized for the presentation of antigens. DCs phagocytose and degrade the microorganism and present $C$. albicans antigens to the membranes bound to the major histocompatibility complex II. This, coupled with the release of cytokines, aids in the activation of naive T cells. $C$. albicans presents several mechanisms to escape from the immune system and to be able to grow and proliferate in the host. One of these is the formation of hyphae. If DCs phagocytose yeast cells, they induce Th1 proliferation, which contributes to fungus elimination. However, Tregs contribute to the elimination of IL-2, which prevents the inhibitory role of this cytokine on the Th17 population, consequently improving the immune response.

Chang et al. (31) found that, compared to non-septic patients, septic patients tended to show persistently lower intracellular production of IFN-y and IL-2 at multiple time-points during the disease course.

The most relevant studies on the various clinical forms of Candida spp. are summarized in Table 1 (A and B), and each of the four categories is described in detail in the following sections.

\section{Vulvovaginal candidiasis}

Vulvovaginal candidiasis (VVC) is a common disorder in women, characterized by vulvar itching, inflammation of the vaginal wall, and vaginal discharge. It is caused by Candida spp., especially $C$. albicans. Systemic immunity is essential to initiate a proper defense against vaginal mucosal Candida infection; however, there are considerable discrepancies among studies investigating the roles played by local humoral and cellular factors, which mediate protection. Moreover, the mechanisms operating against vaginal C. albicans infection remain largely unknown. De Bernardis et al. (32) used rat models of vaginal Candida infection and analyzed the vaginal $\mathrm{CD} 4 / \mathrm{CD} 8 \mathrm{~T}$ cell ratio; they showed that lymphocytes were involved in adaptive anti-candidal immunity at the vaginal level. Furthermore, both pro-inflammatory and anti-inflammatory cytokines were found in the supernatant of vaginal B cells from infected rats.

In another study, De Bernardis et al. (33) determined which molecules of the fungus could best act as targets for the cells of the immune system. For this, they used rats that they actively immunized intravaginally with two proteins with high antigenic capacity: mannoproteins (MP) and serum amyloid P (Sap). They specifically used recombinant forms of a 65-Kd MP and Sap2. Cholera toxin (CT) was used as the adjuvant in some groups. In rats immunized with MP-65Kd + CT or with Sap $2+$ CT, the fungal burden was reduced by $50 \%$ in the first $48 \mathrm{~h}$ compared to that in control rats. Using only 65 -Kd MP or Sap2, the results were better than in the control group, but worse than for combined immunization with CT. If only CT was used in the immunization, the results were similar to those in the control group. Mutants in which the genes for Sap2 production are removed become avirulent; therefore, for vaccine trials, Sap2 was used because it is one of the key proteins in Candida virulence. In collaboration with an external laboratory, a vaccine based on Sap2-coated virosomes (PEVION7) was obtained. Rats immunized with this vaccine showed IgG and IgA in their vaginal fluids against Sap2 and showed faster recoveries than those of the control rats. When the study was transferred to humans (phase I), the results were also found to be positive and independent of the site of administration of the vaccine (intramuscular or intravaginal).

Other proteins important for the Candida infection process are being tested as vaccines with good results: Hyr1 (which inhibits the innate immune system), Sap2 (which contributes to inflammation), and $\beta$-mannan (a key component of the fungal cell wall involved in processes related to adhesion). A multivalent vaccine with several of these antigens may be more effective in protecting against Candida infections (33).

Wang et al. (34) assessed the local immunity of vaginas in women with vulvovaginitis versus a control group using a questionnaire and bacterial culture of vaginal secretions. IL-2 and IL-4 levels were assessed by ELISA. The levels of IL-2 in patients with VVC were not significantly different from those of the control group. Fan et al. (35) studied samples from women with VVC, cured women, and control subjects to detect levels of IL-2, IL-4, and IFN-y in vaginal lavage fluid. The results showed an increase in these interleukins in the affected groups, demonstrating their role in the pathogenesis of VVC (35).

Chen et al. (36) attempted to ascertain the Th1/Th2 cytokine balance in the vaginas of rats with candidiasis and with different immune statuses. Th1 (IL-2), Th2 (IL-4, IL-10), and transforming growth factor beta 1 (TGF- $\beta 1$ ) cytokine mRNA in the vaginas of the rats was quantified by RT-PCR. The study was performed in 153 female mice inoculated intravaginally with $C$. albicans. The mice were divided into the following groups: estrogen treatment prior to inoculation and normal immunity (group D), non-estrogen treatment and normal immunity (group $\mathrm{H}$ ), estrogen treatment prior to inoculation + immunosuppressive treatment (group $\mathrm{G}$ ), and controls with non-estrogen treatment (group I). Levels of IL-2 and IL-4 were higher in groups D and G than in controls. IL-2 levels decreased a few days after inoculation, although they remained above the IL-2 levels of the control mice. IL-1o levels were also higher in groups D and $\mathrm{G}$ than in controls, and higher in $\mathrm{G}$ than in D. The same trends were also observed for TGF- $\beta 1$ levels. Increases in IL-2 levels are known to be related to an improved response to Candida infection, whereas inhibition of IL-2 secretion is associated with suppressed immunity to Candida. In conclusion, the reaction of the local immune response is more important than systemic immune reaction, and treatment of allergic response in women should be reconsidered (35).

Recent studies support the effectiveness of oral and local probiotic treatment for prevention of recurrent vulvovaginal candidiasis (37). Only some of them could demonstrate the role of IL-2 in these defense mechanisms against $C$. albicans (Fig. 8).

Epithelial cell cultures were used by Niu et al. (38) to assess cytokine production, and scanning electron microscopy (SEM) techniques were used to study the adherence and infectivity of C. albicans. The four cell groups in culture were as follows: a first group with cultured epithelial cells (EC), a second group with epithelial cells cultured with $C$. albicans (EC/CA), a third group with epithelial cells cultured with Lactobacillus crispatus (EC/LC), and a fourth group with epithelial cells cultured with $C$. albicans and $L$. crispatus (EC/CA/LC).

It has been shown that the Th1 response is essential for the host to be able to defend itself against $C$. albicans infection. A decrease in IL-2 and IL-4 production in EC/CA culture was observed; however, in EC/CA/LC culture IL-2 levels were increased and IL-4 levels 


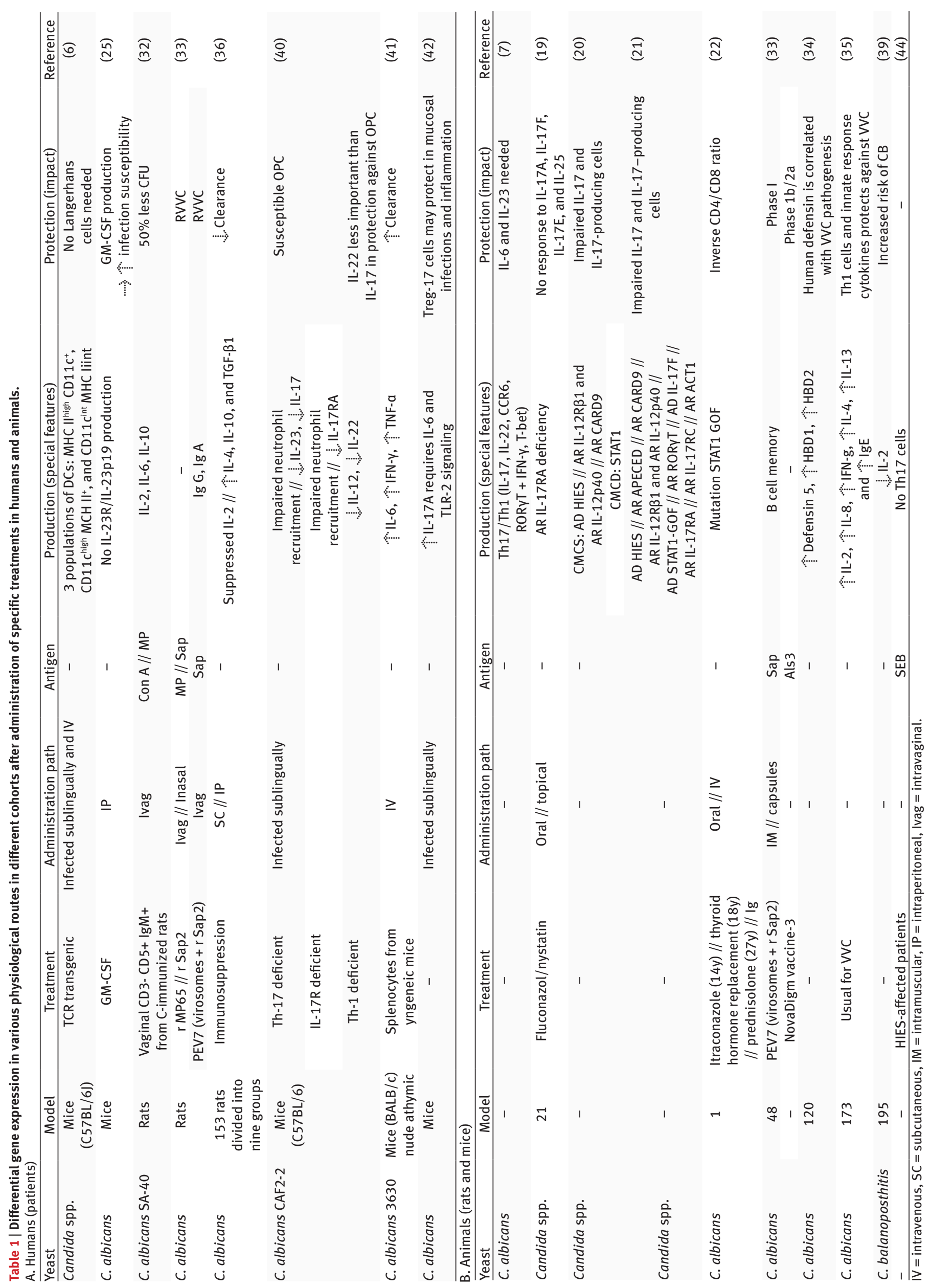




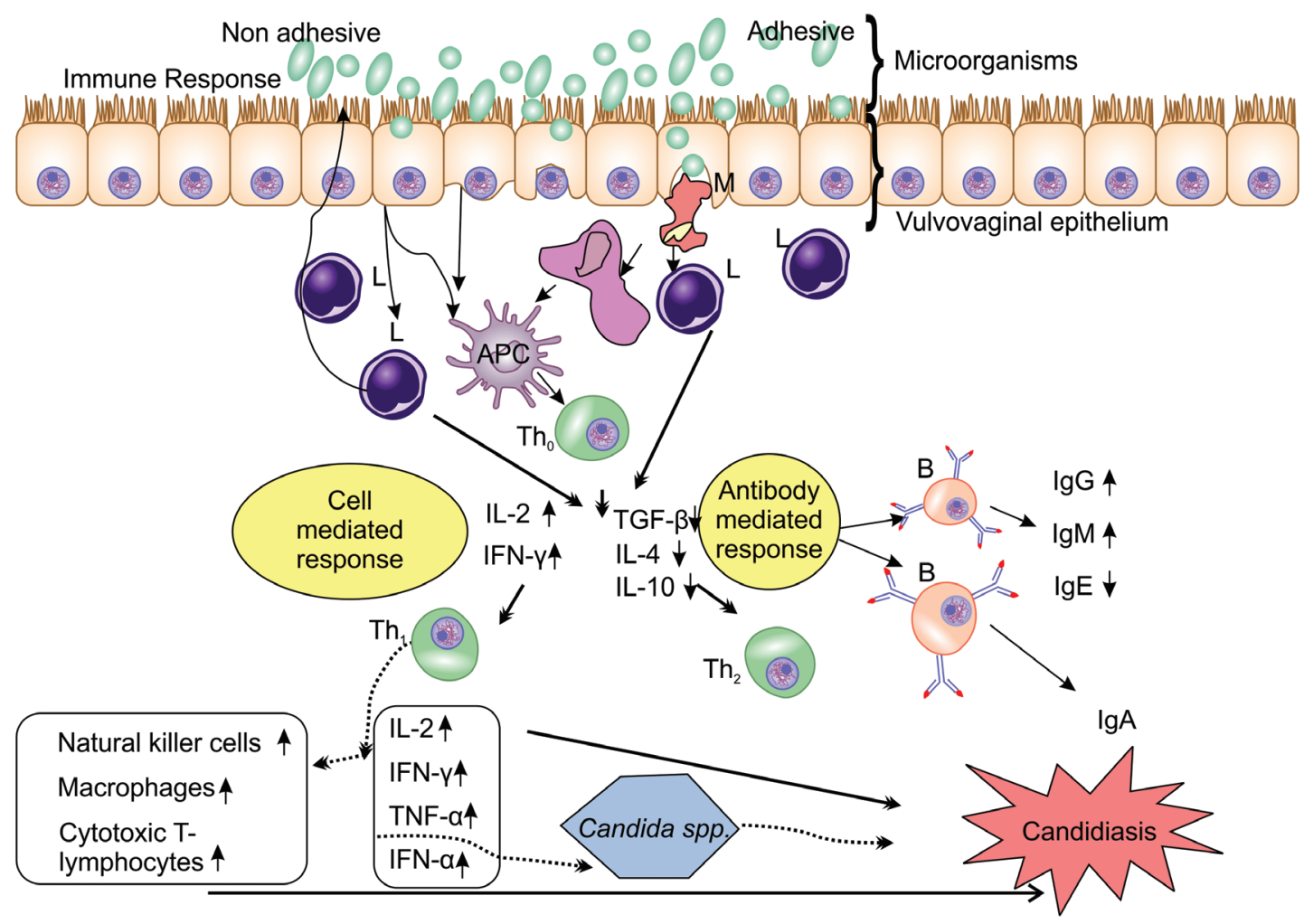

Figure 8 | Probiotics in vulvovaginal candidiasis.

$M=M$ cells of intestinal epithelium, $L=$ lymphocytes, $A P C=$ antigen-presenting cells, $T h=T$ helper cells, IL = interleukin, $T G F=$ tumor growth factor, IFN = interferon, TNF = tumor necrosis factor, Ig = immunoglobulin.

slightly decreased. There was also an increase in IL-6 proinflammatory cytokine levels in EC/CA/LC compared to EC/CA. IL-8 levels decreased in EC/CA/LC compared to EC/CA, coinciding with observations from other studies of an increase in this cytokine during infections. The secretion of IL-17 is also stimulated by L. crispatus, which reduces the viability of $C$. albicans (38).

\section{Candidal balanoposthitis}

Candidal balanoposthitis (CB) is a common condition defined as the inflammation of the foreskin and glans, mainly in uncircumcised males. Balanoposthitis occurs over a wide age range and may have any of several multiple bacterial or fungal origins, the most frequent fungal causal agent being Candida spp. The immune mechanism underlying the disease is being investigated with increasing interest. Chen et al. (39) studied 101 men (6o with recurrent infection and 41 patients with newly diagnosed $C B$ ) for 1 year; 94 healthy men that had not taken immunosuppressive drugs or corticosteroids were used as control subjects. Blood IL-2 concentrations were lower in men with $\mathrm{CB}$ than in the control subjects. The mean IL-2 concentration was $10.5 \mathrm{ng} / \mathrm{ml}$. Subjects were divided into two groups, low IL-2 levels $(<10.5 \mathrm{ng} / \mathrm{mL})$ and high IL-2 levels (>10.5 ng/mL). The low-level group had a higher incidence of CB than the high-level group (70\% vs. 36\%). Other variables were used to discern the relationship with IL-2 levels and the incidence of CB by logistic regression analysis. Low IL-2 levels, comorbidity with other sexually transmitted diseases, and sexual partners with VVC increased the risk of CB in males (39).

\section{Oral and oropharyngeal candidiasis}

Following Conti et al. (40), oropharyngeal candidiasis refers to candidal infection of the oral mucosa. In mice with oral candidiasis infection, Tregs were found to induce IL-17 cytokines in re- sponder T cells, which notably improved fungal clearance and recuperation after infection (26). This suggests that Tregs give rise to acute Th17 cell responses, which consequently intensifies the defense against candidal infections to maintain immune homeostasis. Tregs can drive the change of naive CD4 cells to Th17 cells without the mediation of TGF- $\beta 1$. This is enabled by Tregs consuming IL-2 and enhancing Th17 cell differentiation. Tregs promoted the IL-17A-dependent clearance of fungi during acute candidemia; nevertheless, Tregs could exhibit suppressive properties (40).

However, studies by Gaffen et al. (38) contradicted this theory because they found that Tregs did not induce IL-17 cytokine. They propose that immunity to $C$. albicans depends on multiple immune components, as well as anatomical location. This study shows unequivocally that IL-17 and Th17 cells play a key role in protecting the host against OPC, whereas Th1 cells and IL-22 play a relatively less important role (40).

Farah et al. (41) studied cytokine gene expression in oral tissues of infected euthymic and athymic mice. The mice were orally administered 108 live cells of $C$. albicans. Subsequently, the infection was eliminated and the immune function was also normalized. Interestingly, IL-6, IFN-y, and TNF-a were found in the oral mucosa of both infected and uninfected mice, but IL-2, IL-4, and IL-10 were not detected in euthymic mice. This demonstrates the important role of TNF- $\alpha$ as a mediator in oropharyngeal candidiasis. Bhaskaran et al. (42) showed that, in addition to the increase in Tregs, TLR-2 also promoted IL-17A production by Tregs in oropharyngeal candidiasis, which depended on the cytokine milieu.

Trautwein et al. (43) demonstrated that the most common infected areas are usually the oropharyngeal mucosa and other mucous membranes, as well as the skin. The oropharyngeal mucosa presents cell populations different from those of other mucosa, and so this study uses a model of oropharyngeal candidiasis to show the reactions and interactions between different DC sub- 
populations and $\mathrm{T}$ cell response.

For the study, a transgenic mouse model with Candida-specific TCR was used to inoculate $C$. albicans antigens at the oropharyngeal level to cause infection and examine the antigen presentation and Th17 interaction with DCs. The Candida antigen is presented by the DCs in the lymph node. Candida-derived antigen delivery in cervical lymph nodes is CCR7-dependent. In oral infection, Th17 cells produce IL-17A, IL-17F, and IL-22, whereas IFN-y-producing cells are hardly detected. In contrast, in systemic infection the latter are detected.

Three major populations of DCs in cervical lymph nodes were identified according to MHC II and CD11C expression: population I (MHC IIhigh, CD11C+), population II (CD11chigh, MCH II+), and population III (CD11cint (intermediate level of expression) (int), MHC IIint). Cells from population II were found to capture the antigen from peripheral tissues and the cervical lymph nodes, where they were presented to CD4+ T cells. The cells of population I produce more IL-6 than the other two.

Flt3L-dependent DCs and CCR2-dependent DCs assist in antigen presentation and $\mathrm{T}$ cell priming during oropharyngeal candidiasis. However, Langerhans cells are not necessary for Candida-specific T cell response (43).

\section{Fingernail candidiasis}

According to Milner et al. (44), fingernail candidiasis (FNC) is very common in patients with HIES, who have impaired Th17 cell responses. Ex vivo T cells from subjects with HIES showed a failure to secrete IL-17, but not IL-2, TNF, or IFN-y, upon antigenic stimulation with $C$. albicans. Curiously, patients lacking Tregs, such as those with immune dysregulation, polyendocrinopathy, enteropathy, and X-linked syndrome, which are typified by the progress of several autoimmune disorders, have chronic FNC and other diseases caused by systemic immunosuppression (45).

\section{Prospects of immunotherapy and new therapeutic targets for Candida infections}

New therapeutic targets for Candida infections have recently been developed, taking into account IL-2 expression in candidiasis and its clinical significance. We begin by enumerating the substances that participate in conservation of the microbiota of the vaginal mucosa. L. crispatus has an immunomodulatory effect, inducing cytokine production in epithelial cells. Epithelial cell cultures were used to assess cytokine production, and SEM techniques were used to study the adherence and infectivity of $C$. albicans. The four cell groups in the culture were as follows: cultured epithelial cells (EC), epithelial cells cultured with $C$. albicans (EC/ CA), epithelial cells cultured with L. crispatus (EC/LC), and epithelial cells cultured with $C$. albicans and L. crispatus (EC/CA/LC). SEM demonstrated how $L$. crispatus inhibits the adherence of $C$. albicans to the epithelial cells in culture, improving their resistance to infection. Th1 response is essential for the host to be able to defend itself against $C$. albicans infection. A decrease in IL-2 and IL-4 production in EC/CA was observed; however, EC/CA/LC IL-2 levels increased and IL-4 levels decreased slightly. The secretion of IL-17 is also stimulated by L. crispatus, which reduces the viability of $C$. albicans. Therefore, these results suggested that $L$. crispatus could promote host defense and protect the epithelium from $C$. albicans-induced cell injury; the question here is "are we to believe that the EC synthesized the T cell cytokines in response to C. albicans or L. crispatus, which is the implication here?” (38). According to Datta et al. (46), cytokines/chemokines are also known to mediate the effect of antifungal agents. Polyenes (amphotericin B and nystatin) have been shown to induce the synthesis of pro-inflammatory cytokines; similar immunomodulatory properties have also been observed with azoles and echinocandins in murine models of $C$. albicans infections. These observations provide a theoretical framework for combinatorial treatment approaches, in which the observed antifungal synergy may be partly explained by parallel, synchronous immunomodulation by each therapeutic component (46).

Einsele et al. (47) demonstrated possible methods to obtain specific $\mathrm{T}$ cells against various pathogens that could be transferred to transplant patients for combating opportunistic infections, which would help eliminate the toxicity and side effects of conventionally used drugs. Therefore, it would be of great help to identify the dominant immune-related fungal proteins and peptides that are the targets of $\mathrm{T}$ cells for ultimate therapeutic development and commercialization.

Other relevant mediators of the immune system, according to Tsirigotis et al. (48), are the Janus kinases (JAK), a family of intracellular tyrosine kinases that transduce the cytokine-mediated signal to the nucleus, promoting the expression of certain genes. This route seems to be involved in the resolution of severe sepsis or septic shock by Candida. Future studies need to determine a dose that resolves excessive inflammation without compromising pathogen clearance (48) (Fig. 9).

IL-2 is essential for the development, differentiation, and proliferation of lymphocytes. Their receptors do not have intrinsic kinase activity; therefore, cytoplasmic kinases such as JAK1 or JAK3 are necessary for signal transduction and are therapeutic targets for the treatment of autoimmune diseases (48).

The loss of JAK3 activity is known to result in T lymphocyte dysfunction. The proliferation of these lymphocytes requires continuous exposure to IL-2 for at least $6 \mathrm{~h}$. A recent study claims that the kinase activity of JAK1 is essential and sufficient for phosphorylation of the IL-2-stimulated transcription factor $\mathrm{STAT}_{5}$, but that of JAK3 is dispensable (48). This review suggests the converse. A series of experiments were performed using a JAK3 inhibitor (JA$\mathrm{K} 3 \mathrm{i})$, which acts at the level of the amino acid cysteine, present in

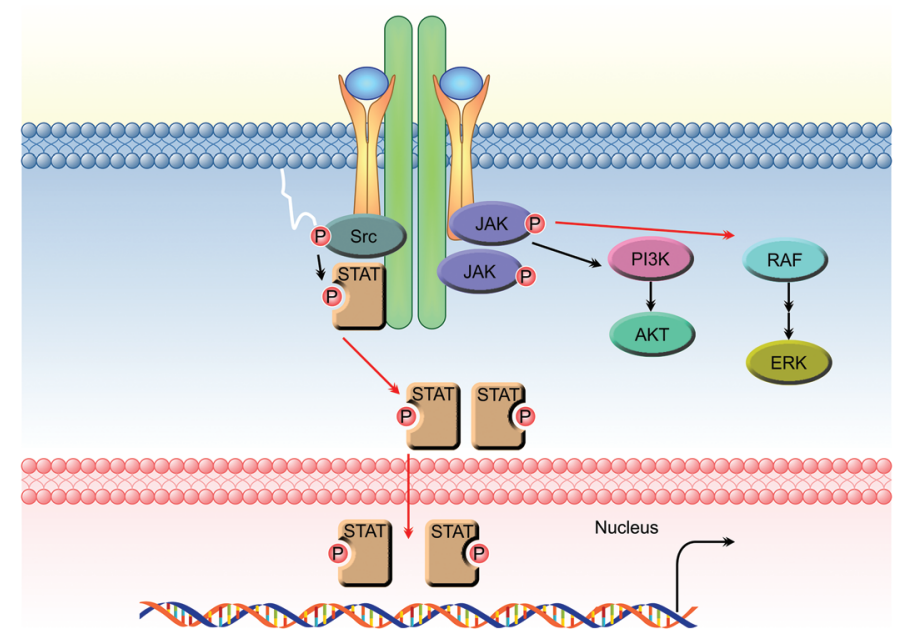

Figure 9 | The recruitment of JAKs induces their phosphorylation, apparently via autophosphorylation or cross-phosphorylation by other JAKs, or by other families of kinases. Activated JAKs then phosphorylate receptors on target tyrosine sites. The phosphorylated receptor sites can serve as docking sites that allow binding of STATs and other SH2-domain-containing signal molecules, such as Src-kinases, Shc, Grb2, and PI3K. 
this type of kinase. In murine cultured CD4+ T lymphocytes, JAK3 was found to be essential for the phosphorylation of STAT 5 after 15 min of stimulation with IL-2 because this phosphorylation did not occur in the presence of JAK3i. In experiments with mutants for JAK3 that lacked cysteine in their structure, the inhibitor did not act, and therefore STAT5 phosphorylation occurred.

By monitoring the activity of STAT 5 for $20 \mathrm{~h}$, a second wave of phosphorylation, not seen in other studies, was found by Smith et al. It indicated that STAT5 phosphorylation peaks within 15 min of IL-2 stimulation and returns to normal levels by $1 \mathrm{~h}$ but subsequently returns to another peak, which it maintains for at least $10 \mathrm{~h}$. By adding JAK3i at different time-points in this second wave, a critical period of 2 to $8 \mathrm{~h}$ after IL-2 stimulation could be determined for $\mathrm{JAK}_{3}$ activity to stimulate the production of certain cyclins that hyperphosphorylate $\mathrm{Rb}$ and lead to DNA replication. The inhibitory effects of JAK3i during the second wave of IL-2 signaling include the suppression of $\mathrm{STAT}_{5}$ and $\mathrm{PI}_{3} \mathrm{~K}$ activities. These effects were tested in vivo in mice given JAK3i and stimulated by IL-2 injections. T lymphocyte proliferation was inhibited in these mice. The second wave of $\mathrm{STAT}_{5}$ phosphorylation produced by IL-2 stimulation showed greater sensitivity to JAK3 inhibition (49).

\section{Conclusion}

Our present review of the literature on the role of IL-2 in host responses to Candida infection suggests that low levels of IL-2 and IFN-y secretion in response to fungal pathogen-produced antigens might substantially account for the deficient cell-mediated immunity observed in patients with fungal infections. This reduction in IL-2 and IFN-y levels might mainly be due to the decrease in Th1 cell numbers. Therefore, modification of cytokine production might be a dominant mechanism contributing to the greater susceptibility to Candida infections in patients suffering from other chronic infections. The lowered capacity to secrete IL-2, IL-17, and IFN-y and the low production of IL-10 suggest that, in these patients, fungal antigens are incapable of promoting sufficient secretion of Th1 cytokines, instead resulting in enhanced secretion of Th2 cytokines.

Additional studies assessing the secretion of other cytokines typical of Th1 or Th2 responses are needed to validate this potential mechanism, and would contribute to the development of new therapeutic approaches, especially for immunocompromised patients.

\section{References}

1. Lionakis SM, Iliev IL, Hohl TM. Immunity against fungi. J Cl Insight. 2017;2: e93156.

2. Manikwar P, Kiptoo P, Badawi AH, Büyüktimkin B, Siahaan TJ. Antigen-specific blocking of $\mathrm{CD}_{4}$-specific immunological synapse formation using BPI and current therapies for autoimmune diseases. Med Res Rev. 2012;32:727-64.

3. Isakson SM, Katzman SD, Hoyer KK. Spontaneous autoimmunity in the absence of IL-2 is driven by uncontrolled dendritic cells. J Immunol. 2012;189:1585-93.

4. Kanehisa M, Furumichi M, Tanabe M, Sato Y, Morishima K. KEGG: new perspectives on genomes, pathways, diseases and drugs. Nucleic Acids Res. 2017;45: D353-61.

5. Mehra P, Wells AD. Long-range transcriptional control of the IL-2 gene by an intergenic enhancer. Mol Cell Biol. 2015;35:3880-91.

6. Miller PG, Bonn MB, McKarns SC. Trans membrane TNF-TNFR2 impairs Th17 differentiation by promoting IL-2 expression. J Immunol. 2015;195:2633-47.

7. Trautwein-Weidner K, Gladiator A, Kirchner FR, Becattini S, Rülicke T, Sallusto F, et al. Antigen-specific Th17 cells are primed by distinct and complementary dendritic cell subsets in oropharyngeal candidiasis. PLoS Pathog. 2015;1:e1005164.

8. Sallusto F, Zielinski CE, Lanzavecchia A. Human Th17 subsets. Eur J Immunol. 2012;42:2215-20.

9. Gil L, Murciano C, Yáñez A, Gozalbo D. Role of Toll-like receptors in systemic Candida albicans infections. Front Biosci (Landmark Ed). 2016;21:278-302.

10. Ju ST, Sharma R, Gaskin F, Fu SM. IL-2 controls trafficking receptor gene expression and Th2 response for skin and lung inflammation. Clin Immunol. 2012;145: 82-8.

11. Whibley N, Mac Caullum DM, Vickers MA, Zafreen S, Waldmann H, Hori S, et al. Expansion of Foxp3+ T-cell populations by Candida albicans enhances both Th17-cell responses and fungal dissemination after intravenous challenge. Eur J Immunol. 2014;44:1069-83.

12. Netea MG, Brown GD, Kullberg BJ, Gow NA. An integrated model of the recognition of Candida albicans by the innate immune system. Nat Rev Microbiol. 2008; 1:67-78.

13. Yamazaki S, Steinman RM. Dendritic cells as controllers of antigen-specific Foxp3+ regulatory T cells. J Dermatol Sci. 2009;54:69-75.

14. Kamiya A, Kikuchi A, Tomita Y, Kanbe T. Epidemiological study of Candida species in cutaneous candidiasis based on PCR using a primer mix specific for the DNA topoisomerase II gene. J Dermatol Sci. 2005;37:21-8.

15. Hattori H, Iwata T, Nakagawa Y, Kawamoto F, Tomita Y, Kikuchi A, et al. Genotype analysis of Candida albicans isolates obtained from different body locations of patients with superficial candidiasis using PCRs targeting $25 \mathrm{~S}$ rDNA and ALT repeat sequences of the RPS. J Dermatol Sci. 2006;42:31-46.

16. Kruger P, Saffarzadeh M, Alexander NR, Weber ANR. Neutrophils: between host defence, immune modulation, and tissue injury. PLoS Pathog. 2015;11: e1004651.

17. Sharma R, Fu SM, Ju ST. IL-2: a two-faced master regulator of autoimmunity. J Autoimmun. 2011;36:91-7.

18. Ryan KR, Hong M, Arkwright PD, Gennery AR, Costigan C, Dominguez M, et al. Impaired dendritic cell maturation and cytokine production in patients with chronic mucocutaneous candidiasis with or without APECED. Clin Exp Immunol.
2008;154:406-14.

19. Lévy R, Okada S, Béziat V, Moriya K, Liu C, Chai LY, et al. Genetic, immunological, and clinical features of patients with bacterial and fungal infections due to inherited IL-17RA deficiency. Proc Natl Acad Sci USA. 2016;113:E8277-85.

20. Puel A, Cypowyj S, Maródi L, Laurent Abel L, Picard C, Casanova J. Inborn errors of human IL-17 immunity underlie chronic mucocutaneous candidiasis. Curr Opin Allergy Clin Immunol. 2012;12:616-22.

21. Okada S, Puel A, Casanova JL, Kobayashi M. Chronic mucocutaneous candidiasis diseases associated within born errors of IL-17 immunity. Clin TransI Immunology. 2016;5:e114.

22. Eslami N, Tavakol M, Mesdaghi M. A gain-of-function mutation of STAT1: a novel genetic factor contributing to chronic mucocutaneous candidiasis. Acta Microbiol Immunol Hung. 2017;64:191-201.

23. Khameneh HJ, Ho AWS, Spreafico R, Derks H, Quek HQY, Mortellaro A. The SykNFAT-IL-2 pathway in dendritic cells is required for optimal sterile immunity elicited by alum adjuvants. J Immunol. 2017;198:196-204.

24. Sancho D, Reis e Sousa C. Signaling by myeloid C-type lectin receptors in immunity and homeostasis. Annu Rev Immunol. 2012;30:491-529.

25. Whitney PG, Bär E, Osorio F, Rogers NC, Schraml BU, Deddouche S, et al. Syk signaling in dendritic cells orchestrates innate resistance to systemic fungal infection. PLoS Pathog. 2014;10:e1004276.

26. Helft J, Anjos-Afonso F, van der Veen AG, Chakravarty P, Bonnet D, Reis e Sousa C. Dendritic cell line age potential in human early hematopoietic progenitors. Cell Rep. 2017;20:529-37.

27. Lilic D, Gravenor I, Robson N, Lammas DA, Drysdale P, Calvert JE, et al. Deregulated production of protective cytokines in response to Candida albicans infection in patients with chronic mucocutaneous candidiasis. Infect Immun. 2003; 71:5690-9.

28. Bahri R, Curt S, Saidane-Mosbahi D, Rouabhia M, et al. Normal human gingival epithelial cells sense $C$. parapsilosis by toll-like receptors and module its pathogenesis through antimicrobial peptides and proinflammatory cytokines. Mediators Inflamm. 2010;2010:40383.

29. Khosravi AR, Shokri H, Darvishi S. Altered immune responses in patients with chronic mucocutaneous candidiasis. J Mycol Med. 2014;24:135-140.

30. Richardson JP, Moye DL. Adaptive immune responses to Candida albicans infection. Virulence. 2015;6:327-37.

31. Chang K, Svabek C, Vazquez-Guillamet C, Sato B, Rasche D, Wilson S, et al. Targeting the programmed cell death 1: programmed cell death ligand 1 pathway reverses $T$ cell exhaustion in patients with sepsis. Crit Care. 2014;18:R3.

32. De Bernardis F, Santoni G, Boccanera M, Lucciarini R, Arancia S, Sandini S, et al. Protection against rat vaginal candidiasis by adoptive transfer of vaginal $B$ lymphocytes. FEMS Yeast Res. 2010;10:432-40.

33. De Bernardis F, Arancia S, Sandini S, Graziani S, Norelli S. Studies of immune responses in Candida vaginitis. Pathogens. 2015;4:697-707.

34. Wang W, Di W, Liao QP, LiuZH, Zhang N, Zhang HY, et al. Study on vaginal produc tion of human defensins and the correlated pathogenetic factors of vulvovaginal candidiasis. Zhonghua Fu Chan Ke Za Zhi. 2008;43:486-9. 
35. Fan SR, Liao QP, Liu XP, Liu ZH, Zhang D. Vaginal allergic response in women with vulvovaginal candidiasis. Int Gynaecol Obstet. 2008;101:27-30.

36. Chen S, Li S, Wu Y, Liu Z, Li J. Local expression of vaginal Th1and Th2 cytokines in murine vaginal candidiasis under different immunity conditions. J Huazhong Univ Sci Technolog Med Sci. 2008;28:476-9.

37. Happel AU, Jaumdally SZ, Pidwell T, Cornelius T, Jaspan HB, Froissart R, et al. Probiotics for vaginal health in South Africa: what is on retailers' shelves? BMC Womens Health. 2017;17:7.

38. Niu XX, Li T, Zhang X, Wang SX, Liu ZH. Lactobacillus crispatus modulates vaginal epithelial cell innate response to Candida albicans. Chin Med J (Engl). 2017;130:273-9.

39. Chen J, Zhou YX, Jin XD, Chen SW. Expression of interleukin-2in candidal balanoposthitis and its clinical significance. Chin Med J (Engl). 2011;124:2776-8.

40. Conti HR, Shen F, Nayyar N, Stocum E, Sun JN, Lindemann MJ, et al. Th17 cells and IL-17 receptor signaling are essential for mucosal host defense against oral candidiasis. J Exp Med. 2009;206:299-311.

41. Farah CS, Gotjamanos T, Seymour GJ, Ashman RB. Cytokines in the oral mucosa of mice infected with Candida albicans. Oral Microbiol Immunol. 2002;17:375-8.

42. Bhaskaran N, Cohen S, Zhang Y, Weinberg A, Pandiyan P. TLR-2 signaling promotes IL-17A production in $\mathrm{CD}_{4}+\mathrm{CD}_{25}+\mathrm{Foxp}_{3}+$ regulatory cells during oropharyngeal candidiasis. Pathogens. 2015;4:90-110.
43. Trautwein-Weidner K, Gladiator A, Kirchner FR, Becattini S, Rülicke T, Sallusto F, et al. Antigen-specific Th17 cells are primed by distinct and complementary dendritic cell subsets in oropharyngeal candidiasis. PLoS Pathog. 2015;11:e1005164.

44. Milner JD, Brenchley JM, Laurence A, Freeman AF, Hill BJ, Elias KM, et al. Impaired $T(H) 17$ cell differentiation in subjects with autosomal dominant hyper-IgE syndrome. Nature. 2008;452:773-6.

45. Puel A, Cypowyj S, Bustamante J. Chronic mucocutaneous candidiasis in humans with inborn errors of interleukin-17 immunity. Science. 2011;332:65-8.

46. Datta K, Hamad M. Immunotherapy of fungal infections. Immunol Invest. 2015 ; 44:738-76

47. Einsele H, Loffler J, Kapp M, Rasche L, Mielke S, Grigoleit UG. Immunotherapy for viral and fungal infections. Bone Marrow Transplant. 2015;50:S51-4.

48. Tsirigotis P, Papanikolaou N, Elefanti A, Konstantinou P, Gkirkas K, Rontogianni $D$, et al. Treatment of experimental Candida sepsis with a Janus kinase inhibitor controls inflammation and prolongs survival. Antimicrob Agents Chemother. 2015;59:7367-73.

49. Smith GA, Uchida K, Weiss A, Taunton J. Essential biphasic role for JAK3 catalytic activity in IL-2receptor signaling. Nat Chem Biol. 2016;12:373-9. 